\title{
Information Flow and the Quality of Scientific Production
}

This is the first edition of Amadeus International Multidisciplinary Journal, a new open acess journal, which has the purpose to motivate and leverage the scientific production of researchers, professors and academics from various fields of knowledge.

The speed of Science and circulation of information, impact the interactions that occur between the actors involved. It is through instruments like this, that new changes tend to happen. The purpose is to facilitate people's lives by improving their understanding of the world as well as elucidating issues before obscure.

The studies based in theoretical foundation, tend to support new knowledge, an endless succession of revelations. The quality of our life as living beings, depends a lot of science, where the processes of interaction and information are directly associated.

With regard to methods and their application, these shall be charged to researchers, professionals prepared in this craft. The release of such papers in scientific journals, behind the possibility of evaluation of it by the scientific community, always attentive to innovations and greedy for maintain the culture of a higher quality in the works disseminated to the public. It is an ethical commitment, which has remained as a tradition among scholars.

Another aspect of this type of dissemination of scientific information, is its ability to interact with new and different media with great potential for dissemination on the web, including through so-called social networking. Therefore it is a great responsibility of an instrument as a scientific journal, before the commitment that needs to have with the truth, with the technical care and the ethics from the information that will be delivered to the public.

Thinking about that, the Periodicals propose a peer review. That is, the evaluation of a scientific work is conducted by two experts: on one side the scientific editor and on the other the evaluator. It is the first function check the beginning and the end of the evaluation process supporting the second, as the constant development of numerous scientific activities in the academy, have led to new problems and new challenges regarding on maintaining the integrity of scientific research. In this sense, new 
instruments and tools such as codes of ethics, ethics boards and a relative apparatus in terms of programs against plagiarism, operate as control mechanisms by the scientific community, proposing principles and criteria for the manuscripts required to difuse.

This edition features a good variety of themes, from The Importance of Prescribing Exercise Programs in Gyms, by the authors: João Paulo da Silva Maciel, Thiago Cabral de Souza, Raphael Rodrigues Dias, and Elizabeth Alves Silva, education and health professionals, who seek to clarify through numbers, the importance of prescribing exercise programs in gyms, in order to improve not only performance, but the quality of life of individuals. Another article, also in the health area is The Musculoskeletal Difficulties in Dental Professionals, by the authors:. Livia Maria dos Santos Landim, et al, bringing current issues and relevant to occupational health.

The article by the Professor and Researcher at the Department of Management of the São Francisco Valley University - UNIVASF, Fernanda Roda de Souza Araújo Cassundé, Nildo Ferreira Cassundé Júnior and Gislene Farias de Oliveira, Guides us on Teaching Research Methods in Management Course: Can We Go Beyond the Survey? A classic subject in the universities and with a high academic value.

The Professor and Researcher Geraldo Carreiro de Barros Filho and Gislene Farias de Oliveira, contemplates ourselves with the comment: Reflections on the Discipline of Psychology in Law Courses. The authors develops the issue with a lot of creativity, including escaping slightly from the academic rigor by bringing a more natural language and less far-fetched to treat the subject.

Finalizing this edition,the article Life and Work of Health Professionals, a rich material on stress and its impact on quality of life of health professionals, by Sidney Medeiros de Oliveira and Vânia Barbosa do Nascimento.

Thus, we invite readers to appreciate the material with a critical eye that enables any possible contributions.

Gislene Farias de Oliveira ${ }^{1}$

Editor in Chief

${ }^{1}$ Psychology (1983) and PhD in Social Psychology from the Federal University of Paraíba (2008). Postdoctoral Fellow in Health Sciences, Faculty of Medicine of ABC / SP (2014). http://lattes.cnpq.br/5084510934957372. Contact: gislenefarias@gmail.com. 